\title{
FACIAL FEATURE ORIENTED BANAN FILTERS
}

\author{
Abdelatif Hussein A.ALI \\ High Institute of Computers and Information Technology, \\ Computer Dept., El-Shorouk Academy, Cairo, Egypt, \\ E-mail: aabouali@hotmail.com
}

\begin{abstract}
Face recognition is an active research area because of the importance of its application areas. Face recognition as almost all image processing based systems begin with acquired images elementary processing. The elementary processing is to enhance and/or highlight features. The elementary processing phase sets up ceiling on the recognition ability since any lost feature cannot easily recovered in consequent steps. Also, false features add ambiguities to the problem and may lead to wrong conclusions. This study proposes basic filters oriented towards face feature extraction. The proposed filters are basic natural nonsquare, BANAN, directive-symmetric filters. So, at each typical pixel point the filters provide four readings (North, South, East, and West).The readings measure the divergence from the point of study between the interior and the exterior in the specified direction. The filters experimental results show competitive performance compared to the Sobel and the Prewitt filters.
\end{abstract}

KEYWORDS: Face Recognition; Biometric Identification; Feature extraction; Surveillance; still image; Image processing; Derivative filters; Face Detection.

\section{INTRODUCTION}

"Face Recognition" is an active area in Computer Vision and Biometrics fields. Face recognition technologies significantly impact security, robotics, human-computer-interfaces, digital cameras, games, entertainment, authentication and image indexing applications. It is one of the most visible and challenging problems in computer vision and pattern recognition. Face detection and recognition is anonymous by nature. The digital image is not matched against a database of known individuals. In contrast, face detection and recognition is the science of facial features finding and recognizing. The problem has been extensively studied in the past two decades [1-12], many representative methods such as: Eigenface [2], Fisherface [1], 3-D based methods [13], and many others have been proposed. The challenges in face recognition include scale, illumination, pose, occlusion, clutters, orientation, computational complexity, and expression variations. To deal with the challenges in practical face recognition system, active shape model and 


\section{FACIAL FEATURE ORIENTED BANAN FILTERS}

active appearance model were developed for face alignment; LBP [14] and its variants were used to deal with illumination changes [6] and probabilistic local approach [9] were proposed for face recognition with occlusion. Although much progress has been made, robust face recognition is still challenging issue. The recognition of a query face image is usually accomplished through two phases: image processing for feature extraction followed by classification process. The most popular classifier includes the nearest neighbor (NN) classifier due to its simplicity and efficiency. In order to overcome NN's limitation that only one training sample is used to represent the query face image, $\mathrm{Li}$ and $\mathrm{Lu}$ proposed the nearest feature line (NFL) classifier [15], which uses two training samples for each class to represent the query face. Chien and $\mathrm{Wu}$ [16] proposed the nearest feature plane (NSP) classifier, which uses three samples to represent the test image. Later on, classifiers using more training samples for face representation were proposed, such as the local subspace classifier (LSC) [18] and the nearest subspace (NS) classifiers, which represent the query sample by all the training samples of each class. The use of the neural networks and Genetic algorithms in face recognition has taken much of interests from the researchers [19-24].

In the search for facial features many methods are considered such as: edge detection, transformations, templates, and moments. The edge detection is the closest to our study however it is not the aim of the study to find edges but rather highlight facial features for further processing. Edge detection techniques attempt to capture discontinuities in photometrical, geometrical and physical characteristics of the objects. An edge detector takes an enhanced image to produce edges with attributes like: position, strength, orientation, and scale. Detectors could process the image with a priori knowledge of the scene and about the edge to be detected or without. The process of edge finding includes core processing step such as: differential filters and labeling. The edge definition cannot be easily inferred from that basic processes but rather requires an extensive search to find out the edges properties and avoid false edges. From the edge detectors techniques, Marr-Hildreth, Roberts Cross, Canny method, The Local Threshold and Boolean Function Based, Color Edge Detection Using Euclidean Distance and Vector Angle, Color Edge Detection using the Canny Operator, and Depth Edge Detection using Multi-Flash Imaging [2728]. 
The shortage of features extraction phase definitely limits the ability and sets a boundary on the final outcome of the system query. The first phase of the process, normally, is a set of filters to extract/ highlight the features of the face. In this study, face oriented filters are proposed. The proposed filters are basic natural non-square, BANAN, directive-symmetric filters. At each typical pixel point the filters provides four readings (North, South, East, and West).The filters measures the divergence between the interior and the exteriors from the reference pixel in the specified direction. The study is confined to the proposal and performance comparison of the filters (operators) with the well-known one's through applying widely used segmentation methods. The proposed filters provide a core data that could be used in the face detection and recognition process.

The remaining of this paper organized as following: Section 2 presents the proposed filters. Section three is a comparative study with the Prewitt and Sobel filters. Multiplicity of operators is pointed out in section 4. Finally, the conclusion of the study is in section 5 .

\section{PROPOSED FACIAL FILTER}

The proposed BANAN filters operates in the four basic directions, North, South, East, and West, from the pixel under study. Figure (1) shows the four filters. The position of the point under computation is marked by the underline. The filters measure the directional difference between the interior and the exteriors of the region next to the point in the specified direction. The four filters are a rotational version of each other's at right angles. To be more specific let us assume that the point under computation is at coordinates $(x, y)$, the origin is the top-left and the direction of the $\mathrm{y}$ is the east and the direction of the $\mathrm{x}$ is the south direction then the operators for that typical pixel are governed by the following equations:- 


\section{FACIAL FEATURE ORIENTED BANAN FILTERS}

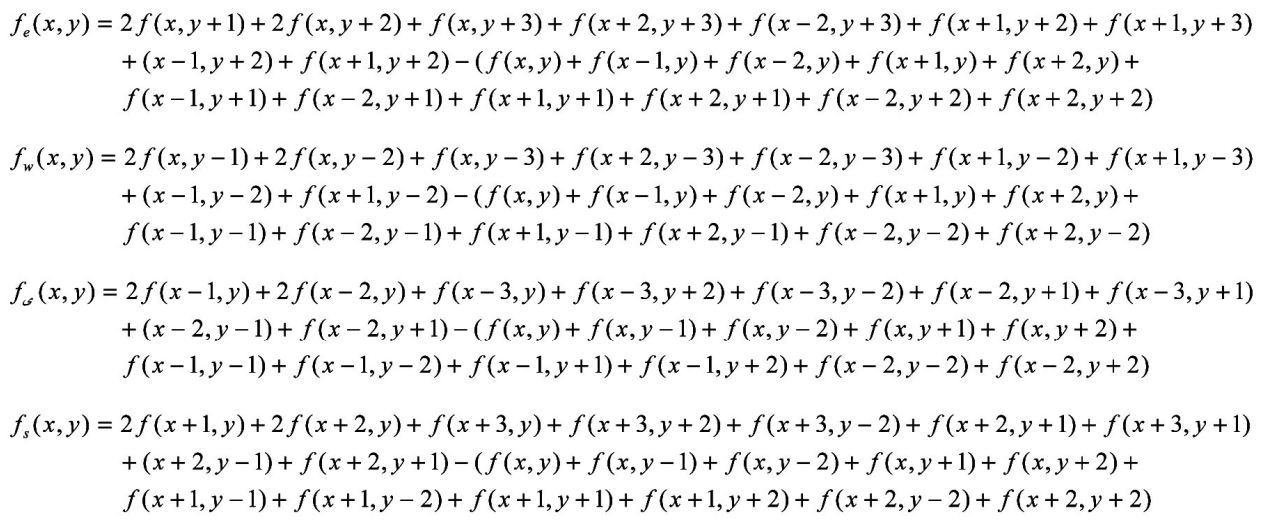

\begin{tabular}{|l|l|l|l|}
\hline 1 & -1 & -1 & -1 \\
\hline 1 & 1 & -1 & -1 \\
\hline 1 & 2 & 2 & $\mathbf{- 1}$ \\
\hline 1 & 1 & -1 & -1 \\
\hline 1 & -1 & -1 & -1 \\
\hline
\end{tabular}

West

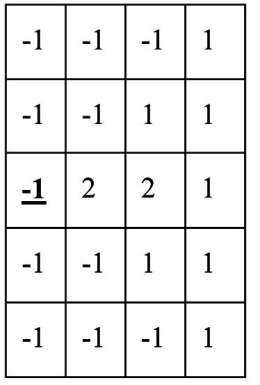

East

\begin{tabular}{|l|l|l|l|l|}
\hline 1 & 1 & 1 & 1 & 1 \\
\hline-1 & 1 & 2 & 1 & -1 \\
\hline-1 & -1 & 2 & -1 & -1 \\
\hline-1 & -1 & $\underline{-1}$ & -1 & -1 \\
\hline
\end{tabular}

North

\begin{tabular}{|l|l|l|l|l|}
\hline-1 & -1 & $\underline{-1}$ & -1 & -1 \\
\hline-1 & -1 & 2 & -1 & -1 \\
\hline-1 & 1 & 2 & 1 & -1 \\
\hline 1 & 1 & 1 & 1 & 1 \\
\hline
\end{tabular}

South

Figure (1) the four basic directional filters

The filters shape matches most of the face natural cavities. The values given by the filter provides elementary measures to the shape of the surface around the pixel under the study. The four signed values of the filters on regional bases can assist in taking the right decisions for both face detection and recognition phase.

To make our point we will run the filters on standard faces taken from [25]. Figure (2) presents the results of applying the above filters on a face randomly selected from the former mentioned standard library figure (2)-a. Figure (2)-b,c,d represent the measurements of the East positive, negative, and absolute consequently. It is clear from the figure that the filter highlighted the features in the East direction. Figure (2)-e-f-g depicts the same results for the West direction. The same conclusion could be easily 
Journal of the ACS, Vol. 7, May 2013

drawn by visual inspection of the figures. It could be easily noticed that the filter highlighted the same main features of the East one. However there are slight differences could be easily noticed between the two operators. Figure (3) show the results of applying the South, North operators on the same face. The results points to the same conclusion of the above operators.

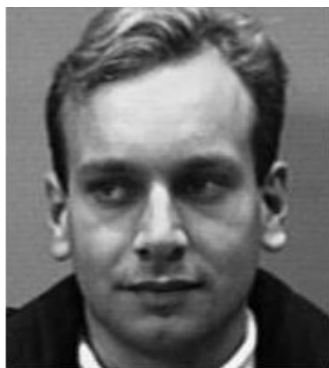

(a) Original

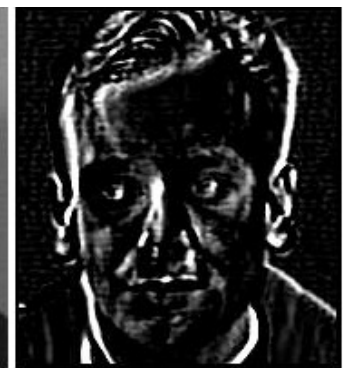

(b) East Positive

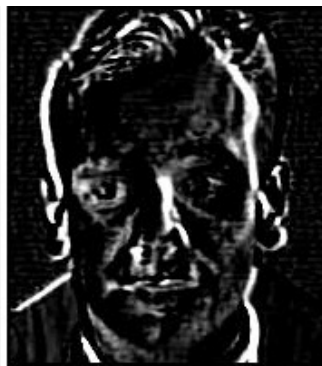

(c) East Negative

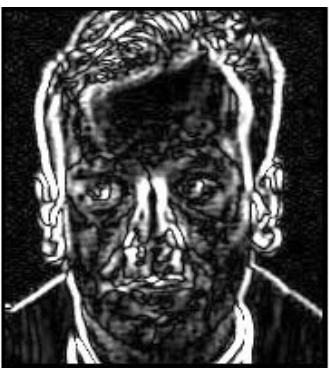

(d) East Absolute

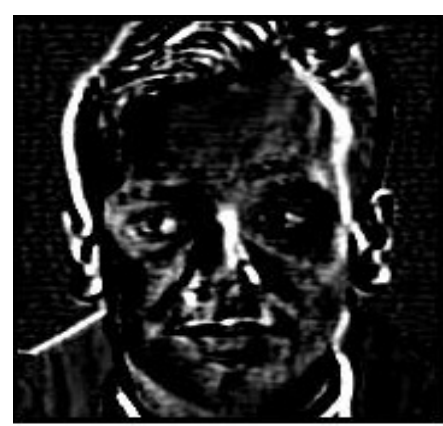

(e) West Positive

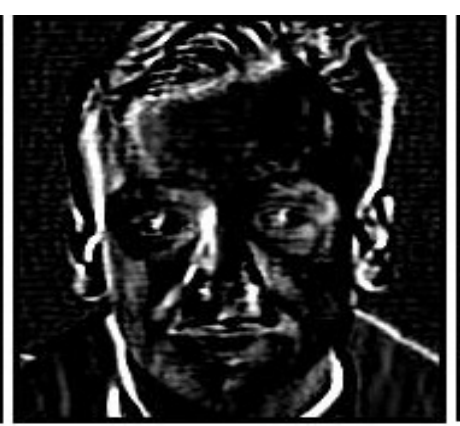

(f) West Negative

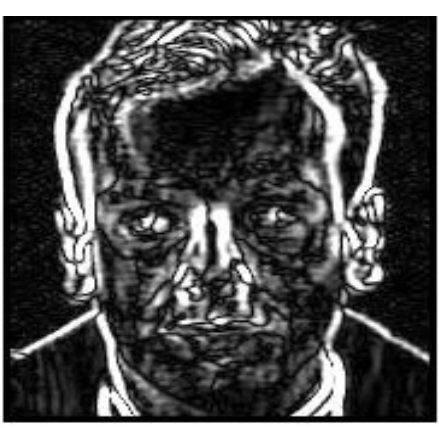

(g) West Absolute

Figure (2) East-West Filters 


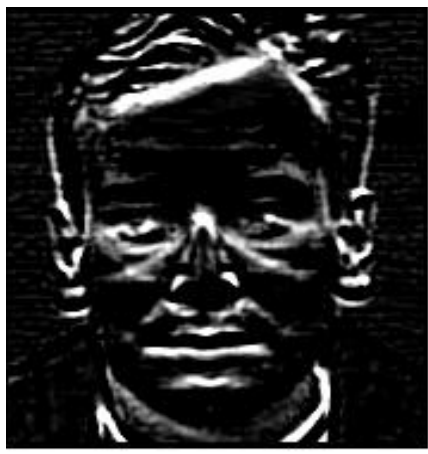

(a) North Positive

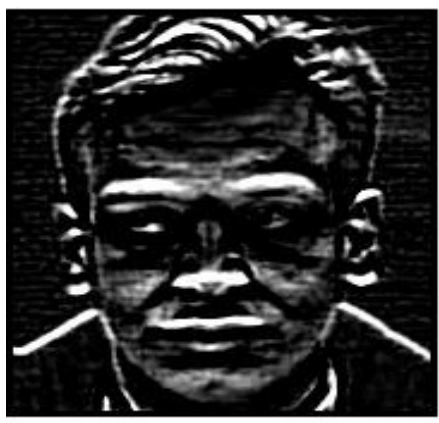

(d) South Positive

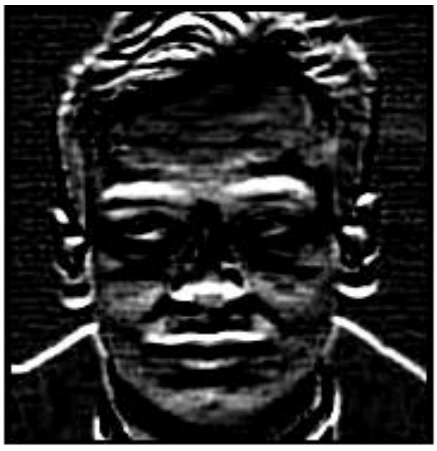

(b) North Negative

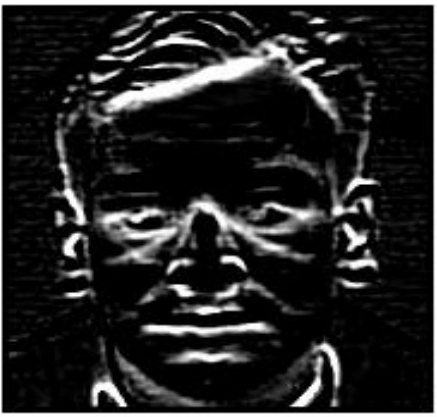

(e) South Negative

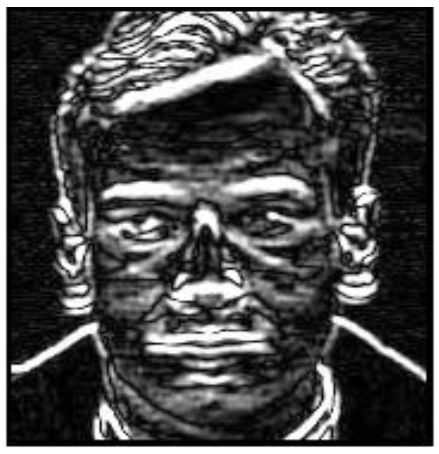

(c) North Absolute

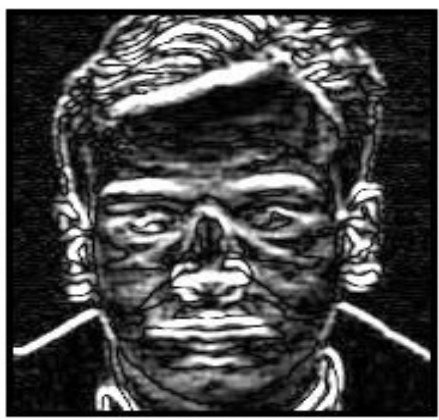

(f) South Absolute

Figure (3) results of North-South operators

Zero-crossing [26] is one of the wildly used methods for edge detection. So, we will consider computation of the zero-crossing after applying the proposed operator. Figure (4) represents the results of zero crossing after the four operators. It could be easily noticed that the process marked out the facial features that could be easily seen as well as that could be hardly noticed in the original image. The zero-crossing results show that there is no significant differences between the opposite directions results, however they are not the same. 


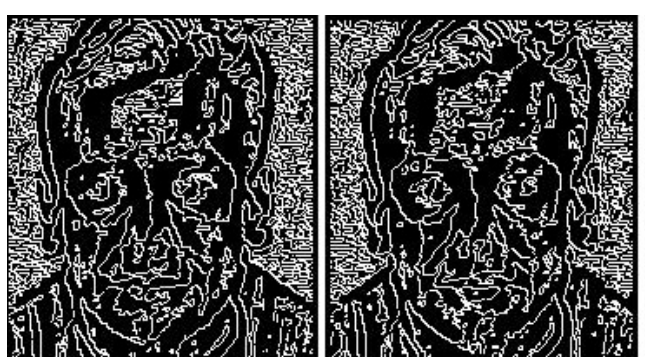

(a) East

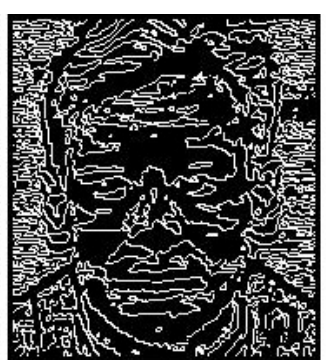

(c) North

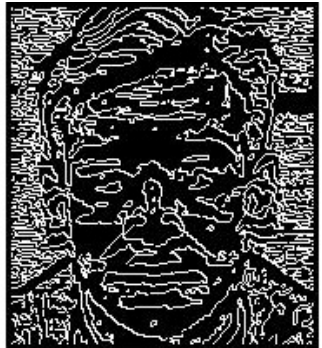

(d) South

Figure (4) Zero Crossing in the four basic directions

Another famous method for segmentation is Otsu's method [26]. Otsu's method searches for the threshold that minimizes the intra-class variance (the variance within the class), defined as a weighted sum of variances of the two classes:-

$$
\sigma_{w}^{2}(t)=\omega_{1}(t) \sigma_{1}^{2}(t)+\omega_{2}(t) \sigma_{2}^{2}(t)
$$

Weights $\omega_{i}$ are the probabilities of the two classes separated by a

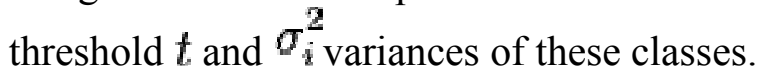

The results of applying the applying Otsu's method on the absolute values of the proposed filters depicted in figure (5).

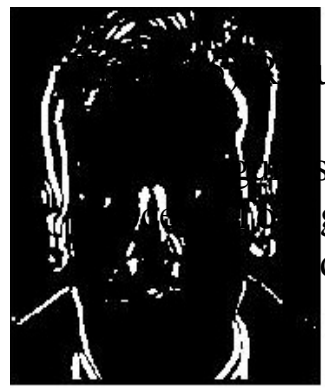

(a) East

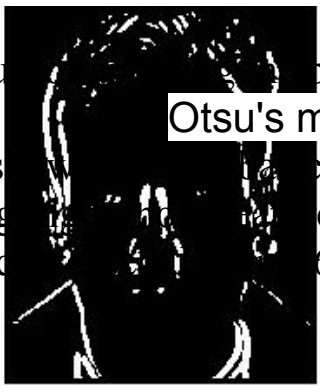

(b) West

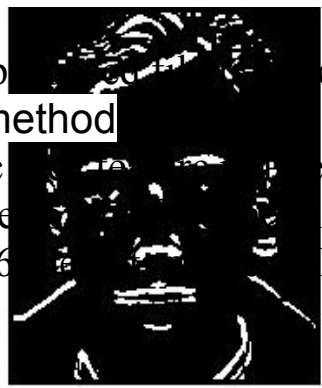

(c) North

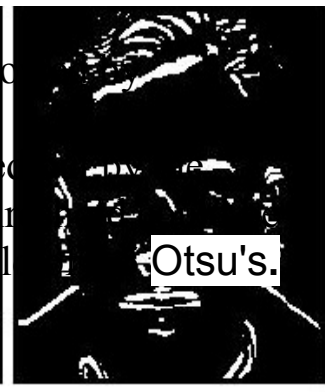

(d) South 


\section{FACIAL FEATURE ORIENTED BANAN FILTERS}

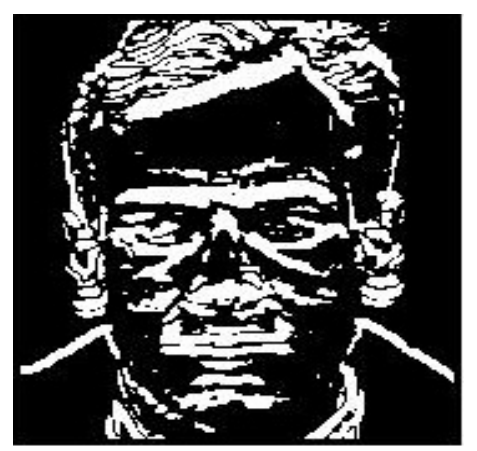

Threshold Based

Figure (6) 0.5 Otsu's threshold

\section{Comparative Study}

This section presents the results of Sobel and Prewitt filters versus the proposed filters using, the segmentation methods mentioned in the previous section, zero-crossing and Otsu's method.

Figure (7)-a original standard image is taken from the library mentioned before. Figure (7)-b, c are Sobl-X,Y consequently. Figure (7)-d ,e are Prewitt-X, Y. Figure (7) -F,G is the proposed North, East. From the figures, we can easily notice that the proposed operators highlighted more features compared to the other two. 


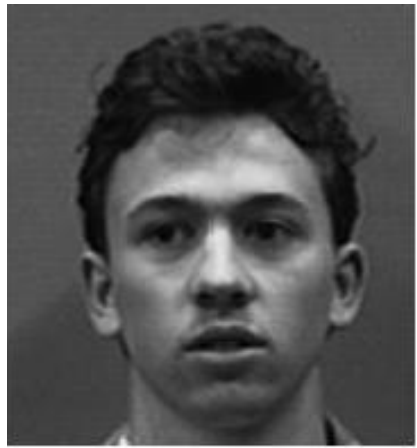

(a) Original

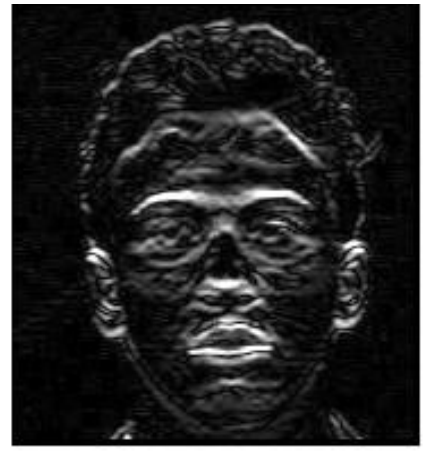

(b) Sobel $x$

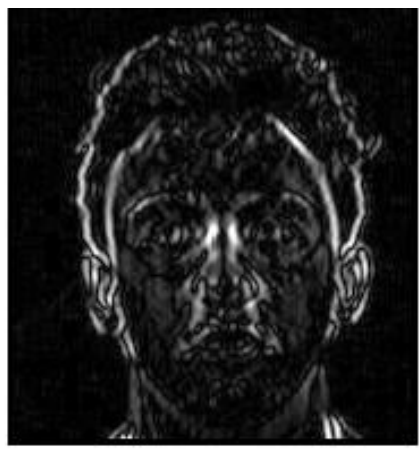

(c) Sobel $Y$

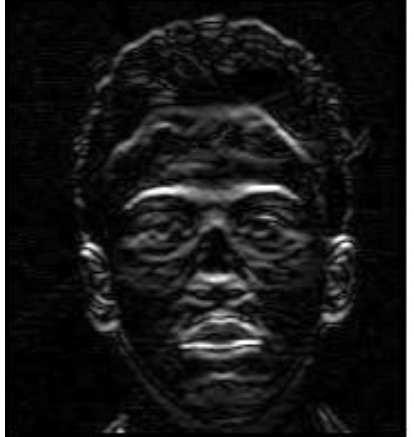

(d) Prewitt $x$

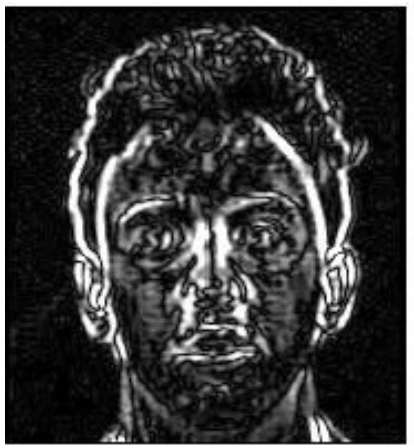

(F) Proposed East

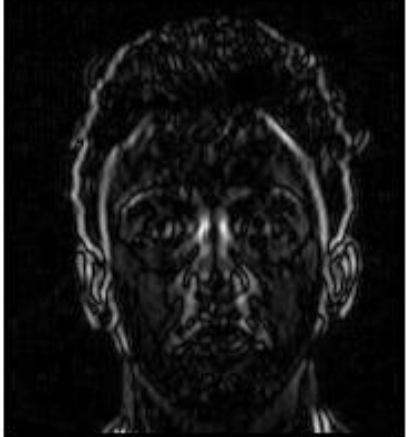

(e) Prewitty

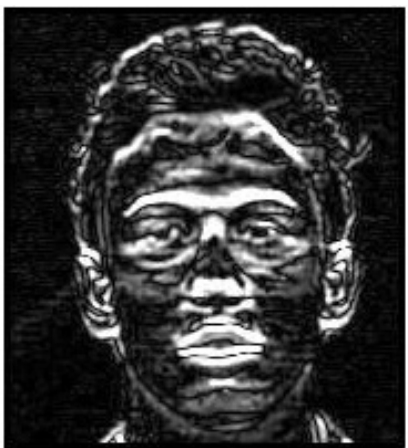

(G) Proposed South

Figure (7) Prewitt, Sobel, and Proposed filters outcomes. 
Figure (8) for the same standard face Otsu' method for Prewitt (a, b), Sobel (c, d), and proposed is (e,f). The results indicate the stability of the detection and the ability to detect more features compared to the others.

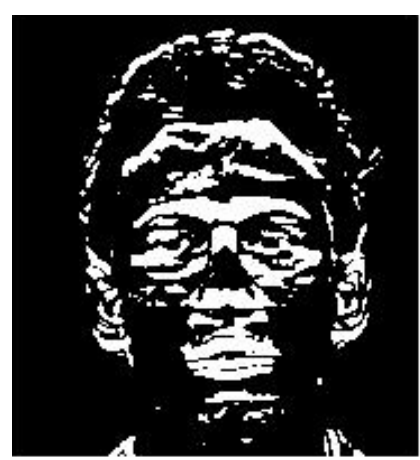

(a) North Proposed

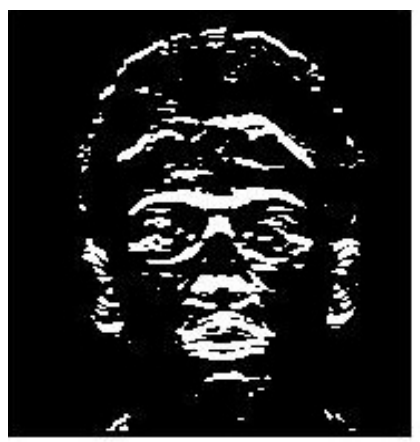

(C) Prewitt $x$

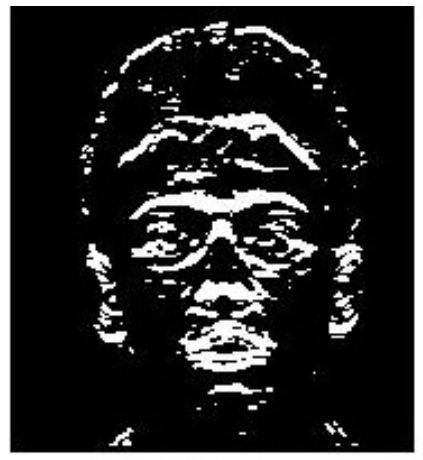

(e) Sobel $x$

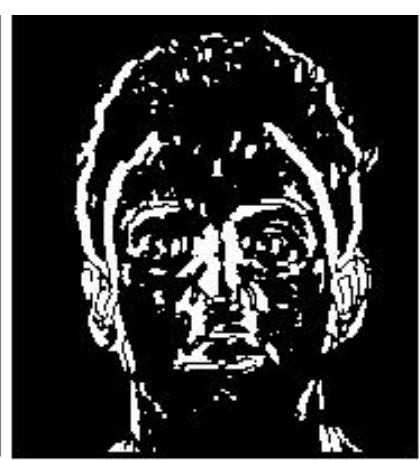

(b) East Proposed

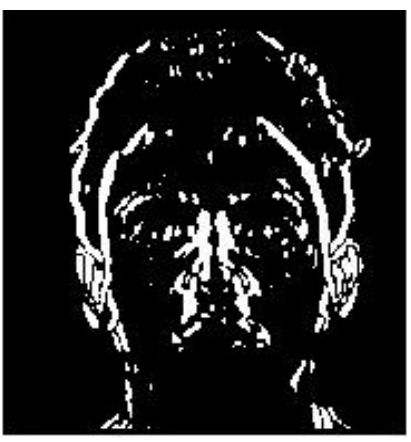

(D) Prewitt Y

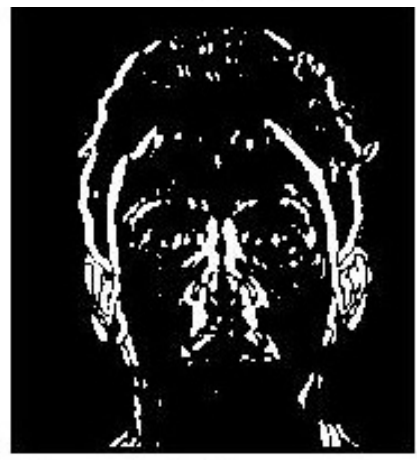

(f) Sobel $Y$

Figure (8) Otsu's method :Prewitt, Sobel, and proposed. 
Figure (9) presents the application of the Zero-Crossing: Sobel $((\mathrm{x}),(\mathrm{y}))$, Prewitt $((\mathrm{x}),(\mathrm{y}))$, and the proposed (east, west, north, south). The results are consistent with the previous ones.

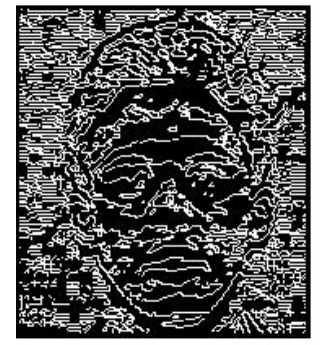

SobelX

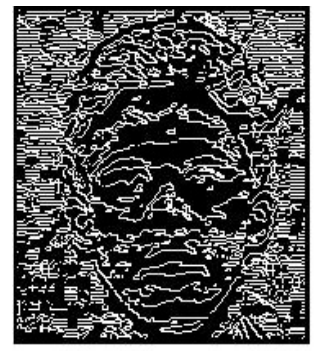

Prewit Zero crossing $X$

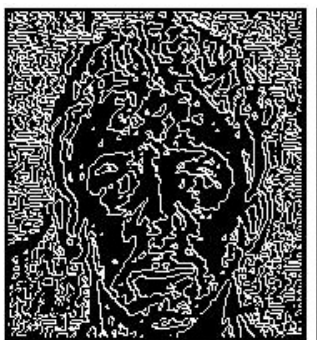

Proposed: East

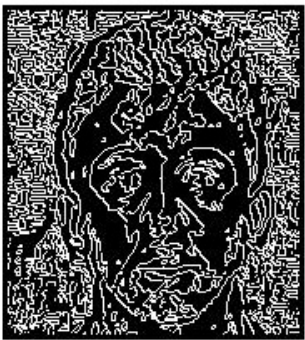

West

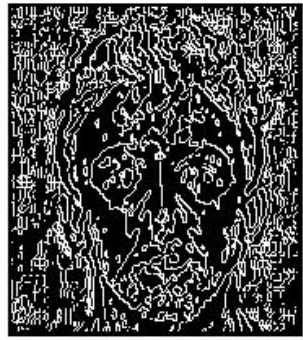

Sobel Y

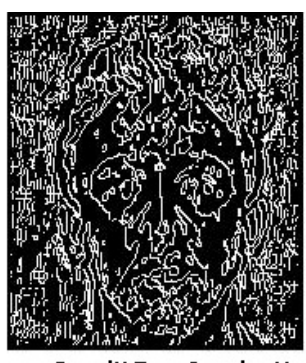

Prewitt Zero Crossing $Y$

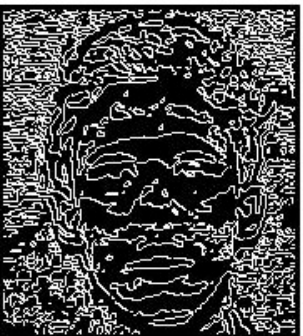

North

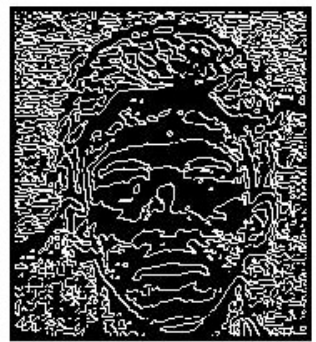

South

Figure (9) Zero-Crossing: Prewitt, Sobel, and proposed.

\section{Multiple operators.}

In this section, we will examine the possibilities of making use of combinations of the proposed operators. We will limit the study to the use of two cases $f_{e}-f_{w}$ and $f_{n}-f_{s}$ which is the divergence in one direction and the negative of divergence of the opposite. The same two methods, Otsu's method and zero-crossing, previously used are considered. Figure(10) 


\section{FACIAL FEATURE ORIENTED BANAN FILTERS}

summaries the results. The figure shows more sold pointing to the face features compared to use of single operator.

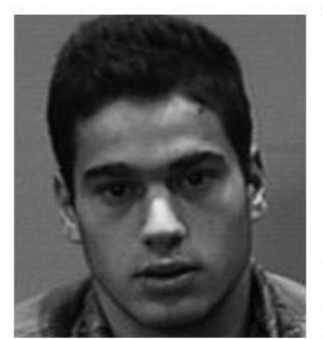

Original

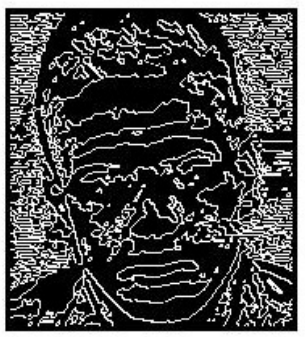

Zero-Crossing North-South

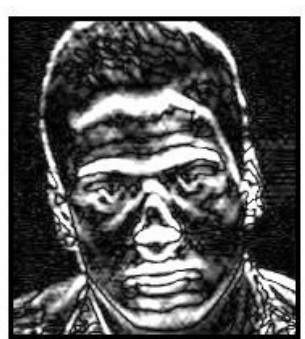

North-South

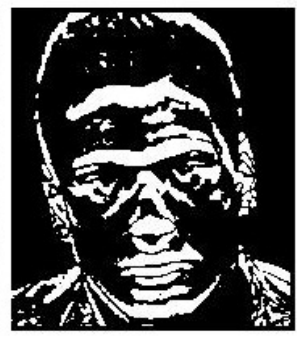

Otsu North-South

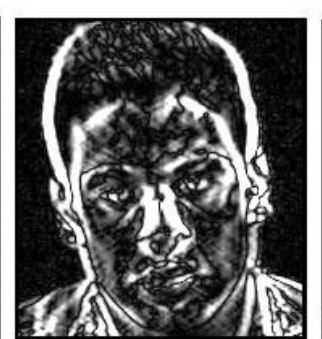

East-West

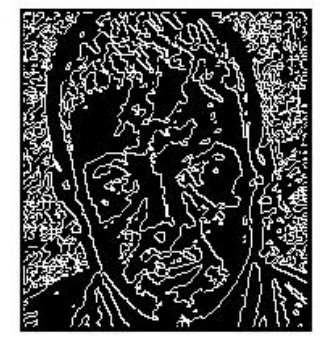

Zero-Crossing East-West

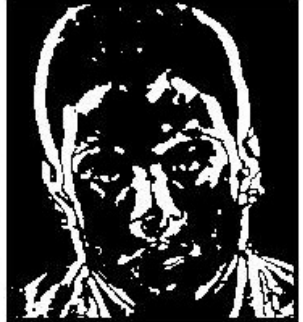

Otsu East-West

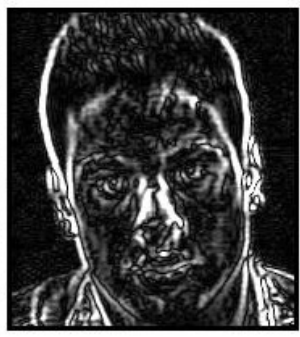

West

Figure (10) North-South operator's results

\section{CONCLUSION}

A proposed basic directional filters presented in this study. The proposed filters are directive and direction-symmetric. The proposed filters provide per pixel four measurements. The measurements are in the four basic directions (north, south, east, and west). The filters measure the divergence between the interior pixels and the exteriors from the reference pixel in the specified direction. The filters experimental results indicated that it is capable of pointing out the facial features. The study included the use of zero-crossing and Otsu's method. The performance of the filters compared in its simplest form with the well-known Sobel and Prewitt filters. In the comparison Otsu's method and the zero crossing are used. The results indicated that the proposed filters have the potential of sold highlighting to face features as well as more stability in its outcome. The results of using multiple operators combined showed improve in the performance compared to single operator. The filters real utilization requires processing of the four values all together over regions in more complicated process. We believe that utilizing the four values could position the key face features accurately as well as answer the questions of: is there a face or not?, and if exist, where? As well as where are the features of that face? 
Journal of the ACS, Vol. 7, May 2013

\section{REFERENCES}

[1] Chellapa, R., Wilson, C. L., and Sirohey, S. Human and machine recognition of faces: A survey. Proc. IEEE,83, 705-740.1995

[2] M Turk and A Pentland, "Eigenfaces for ecognition",Journal of Cognitive Neuroscience, Vo1.3, No. 1, 1991,pp.71-86.

[3] D. L. Swets and J. J. Weng, "Using discriminant eigenfeatures for image retrieval", IEEE Trans. PAMI., vol. 18, No. 8, 831-836, 1996.

[4] C.Magesh Kumar, R.Thiyagarajan, S.P.Natarajan, S.Arulselvi,G.Sainarayanan, "Gabor features and LDA

based Face Recognition with ANN classifier",Procedings Of ICETECT 2011

[5]Önsen TOYGAR Adnan ACAN ,'Face recognition using PCA,LDA and ICA approaches on colored Images", Journal Of Electrical and Electronics Engineering, vol- 13,2003

[6]Y. Cheng, C.L. Wang, Z.Y. Li, Y.K. Hou and C.X. Zhao,” Multiscale principal contour direction for varying lighting face recognition”, Proceedings of IEEE 2010

[7]Issam Dagher,'Incremental PCA-LDA algorithm", International Journal of Biometrics and Bioinformatics (IJBB), Volume (4): Issue (2)

[8]J. Shermina,V. Vasudevan,"An Efficient Face recognition System Based on Fusion of MPCA and LPP”, American Journal of Scientific Research ISSN 1450-223X Issue 11(2010), pp.619

[9] Ishwar S. Jadhav, V. T. Gaikwad, Gajanan U. Patil,'Human Identification Using Face and Voice

Recognition", International Journal of Computer Science and Information Technologies, Vol. 2 (3), 2011

[10] Yun-Hee Han,Keun-Chang Kwak, " Face Recognition and Representation by Tensorbased MPCA

Approach”, 2010 The 3rd International Conference on Machine Vision (ICMV 2010)

[11] Neerja,Ekta Walia, "Face Recognition Using Improved Fast PCA Algorithm", Proceedings of IEEE 2008

[12] Shan S, Yang P, Chen X, Gao W AdaBoost Gabor Fisher classifier for face recognition. In Proceedings of International workshop on analysis and modeling of faces and gestures (AMFG 2005), LNCS 3723, pp 278- 291,2005

[13] Nese Alyuz, Berk Gokberk, and Lale Akarun3-D Face Recognition Under Occlusion Using Masked Projection, IEEE TRANSACTIONS ON INFORMATION FORENSICS AND SECURITY, VOL. 8, NO. 5, MAY 2013

[14] W. Zhang, S. Shan, W. Gao, X. Chen, and H. Zhang, "Local Gabor binary pattern histogram sequence (LGBPHS): A novel non-statistical model for face representation and recognition," in Proc. Int. Conf. Computer Vision (ICCV), 2005, vol. 1, pp. 786-791. 


\section{FACIAL FEATURE ORIENTED BANAN FILTERS}

[15 ]Li, S. Z. and Lu,J. (1999). "Face recognition using the nearest feature line method". IEEE Transactions on Neural Networks, 10(2):439-443.

[16] J.-T. Chien and C.-C. Wu, "Discriminant wavelet faces and nearest feature classifiers for face recognition", IEEE Trans. Pattern Anal. Machine Intell., 24(12):1644-1649, 2002.

[17] W. Zheng, L. Zhao, and C. Zou, "Locally nearest neighbor classifiers for pattern classification", Pattern Recognition,37(6):1307-1309, 2004.

[18] Hotta, Seiji, Local Subspace Classifier with Transform-Invariance for Image Classification IEICE Transactions on Information and Systems, Volume E91.D, Issue 6, pp. 1756-1763 (2010).

[19] Steve Lawrence,C.Lee Giles,A.h Chung Tsoi,Andrew D. Back, ” Face Recognition: A Convolutional

Neural Network Approach"

[20] Lin-Lin Huang, Akinobu Shimizu, Yoshihiro Hagihara, Hidefumi Kobatake,’Face detection from

cluttered images using a polynomial neural network", Elsevier Science 2002

[21] HyoungWoo Lee, SeKee Kil , *Younghwan Han, SeungHong Hong,' Automatic Face and Facial Features Detection', ISIE 2001, Pusan, KOREA.

[22] GhanshyamRaghuwanshi, Nishchol Mishra, Sanjeev Sharma "Content based Image Retrieval using Implicit and Explicit Feedback with Interactive Genetic Algorithm", International Journal of ComputerApplications, Vol 43- No.16, April 2012

[23] Ajoy Kumar Dey, Susmita Saha, Avijit Saha, Shibani Ghosh, "A Method of Genetic Algorithm (GA) for FIR

Filter Construction: Design and Development with Newer Approaches in Neural Network Platform", (IJACSA)

International Journal of Advanced Computer Science and Applications, Vol. 1, No. 6, pp. 87-90, 2010.

[24] Yuuki Yokoo, Masfumi Hagiwara,'Human Faces Detection Method using Genetic Algorithm", Evolutionally Computation, 1996 Proceedings of IEEE International Conference, pp. 113 -1 18

[25] Collection of Facial Images: Faces94, http://cswww.essex.ac.uk/mv/allfaces/faces94.html

[26] R.C.Gonzalez, R.E.Woods Gonzalez, Digital Image Processing, AddisonWesley, 2012.

[27] Ehsan Nadernejad, Edge Detection Techniques:Evaluations and Comparisons, Applied Mathematical Sciences, Vol. 2, 2008, no. 31, 1507 - 1520.

[28] M. A. Berbar, H. M. Kelash, and A. A. Kandeel, "Faces and Facial Features Detection in Color Images",Proc. of IEEE GMAI, Palma De Mallorca, Spain, August, 2006, pp.209-214. 